\title{
Sex differences in the effects of maternal vitamin supplements on mortality and morbidity among children born to HIV-infected women in Tanzania
}

\author{
Kosuke Kawai $^{1} *$, Gernard Msamanga ${ }^{2}$, Karim Manji ${ }^{3}$, Eduardo Villamor ${ }^{1,4,5}$, Ronald J. Bosch ${ }^{6,7}$, \\ Ellen Hertzmark ${ }^{1}$ and Wafaie W. Fawzi ${ }^{1,4,8}$ \\ ${ }^{1}$ Department of Epidemiology, Harvard School of Public Health, 677 Huntington Ave, Boston, MA, 02115, USA \\ ${ }^{2}$ Department of Community Health, Muhimbili University of Health and Allied Sciences, Dar es Salaam, Tanzania \\ ${ }^{3}$ Department of Pediatrics and Child Health, Muhimbili University of Health and Allied Sciences, Dar es Salaam, Tanzania \\ ${ }^{4}$ Department of Nutrition, Harvard School of Public Health, Boston, MA, USA \\ ${ }^{5}$ Department of Society, Human Development, and Health, Harvard School of Public Health, Boston, MA, USA \\ ${ }^{6}$ Department of Biostatistics, Harvard School of Public Health, Boston, MA, USA \\ ${ }^{7}$ Center for Biostatistics in AIDS Research, Harvard School of Public Health, Boston, MA, USA \\ ${ }^{8}$ Department of Global Health and Population, Harvard School of Public Health, Boston, Massachusetts, USA \\ (Received 21 August 2009 - Revised 2 November 2009 - Accepted 14 December 2009 - First published online 9 March 2010)
}

\begin{abstract}
We examined whether there are sex differences in the effect of vitamin supplements on birth outcomes, mortality and morbidity by 2 years of age among children born to HIV-infected women in Tanzania. A randomised placebo-controlled trial was conducted among 959 mother-infant pairs. HIV-infected pregnant women were randomly assigned to receive a daily oral dose of one of four regimens: multivitamins (vitamins B-complex, C and E), vitamin A plus $\beta$-carotene, multivitamins including vitamin A plus $\beta$-carotene or placebo. Supplements were administered during pregnancy and continued after delivery. The beneficial effect of multivitamins on decreasing the risk of low birth weight was stronger among girls (relative risks $(\mathrm{RR})=0.39,95 \% \mathrm{CI} 0.22,0.67)$ than among boys $(\mathrm{RR}=0.81,95 \% \mathrm{CI} 0.44,1.49 ; P$ for interaction $=0.08)$. Maternal multivitamin supplements resulted in $32 \%$ reduction in mortality among girls ( $R R=0.68,95 \%$ CI $0.47,0.97$ ), whereas no effect was found among boys $(\mathrm{RR}=1.20,95 \% \mathrm{CI} 0.80,1.78 ; P$ for interaction $=0.04)$. Multivitamins had beneficial effects on the overall risks of diarrhoea that did not differ by sex. Vitamin A plus $\beta$-carotene alone increased the risk of HIV transmission, but had no effects on mortality, and we found no sex differences in these effects. Sex differential effects of multivitamins on mortality may be due to sex-related differences in the immunological or genetic factors. More research is warranted to examine the effect of vitamins by sex and better understand biological mechanisms mediating such effects.
\end{abstract}

Vitamin A: Multivitamins: Sex: Child mortality: HIV

Vitamin A supplementation in children aged 6 months to 5 years has been shown to reduce mortality by $24-30 \%^{(1-3)}$. However, benefits for supplementing young infants less than 6 months of age have been inconclusive ${ }^{(4)}$; Benn et al. ${ }^{(5)}$ speculated that the lack of beneficial effects could be due to differences in the effect of supplements by sex. Three previous trials found that neonatal vitamin A supplementation may have a beneficial effect on mortality in boys but no effect in girls ${ }^{(6-8)}$. A recent study has found that sex differences in the effects of vitamin A on mortality depend on the different dosages of vitamin A, and a lower dosage may be beneficial among girls ${ }^{(9)}$. Studies that examine sex differential effects of other vitamins are still scarce. The possible mechanisms are not understood, but they could be due to sex-related differences in the developing immune system or the degree of micronutrient deficiencies by $\operatorname{sex}^{(10)}$. It has also been hypothesised that vitamins may enhance the effect of the non-specific immune modulation induced by live vaccines, which may have sex differential survival effects ${ }^{(11)}$.

Children born to HIV-infected women are at a high risk of mortality; however, no studies have examined sex differential effects of vitamin supplement among children born to HIVinfected mothers. Nearly, 2 million children were infected with HIV and 270000 died of AIDS worldwide in $2007^{(12)}$. Almost $90 \%$ of all HIV-infected children live in sub-Saharan Africa. We have previously reported that maternal multivitamin supplements showed no effect on overall mortality among children born to HIV-infected mothers in Tanzania ${ }^{(13)}$. Vitamin A plus $\beta$-carotene alone increased the risk of vertical HIV transmission. In the present paper, we examined whether there are sex differences in the effect of maternal supplementation of multivitamins or vitamin A plus $\beta$-carotene on birth outcomes, mortality and morbidity among children born to HIV-infected mothers.

Abbreviation: RR, relative risks.

*Corresponding author: Kosuke Kawai, fax +1617566 7805, email kkawai@hsph.harvard.edu 


\section{Methods}

\section{Study design and population}

From April 1995 to July 1997, 1078 HIV-infected pregnant women were enrolled in a randomised, double-blind, placebo-controlled trial at four prenatal clinics in Dar es Salaam, Tanzania. Details of the study design have been published $^{(13-15)}$. In brief, women were eligible if they were HIV infected, pregnant between 12 and 27 weeks' gestation age at enrolment, resided in Dar es Salaam and had consented to participate in the trial. We tested HIV-1 serostatus by ELISA (Wellcozyme, Murex Biotech Ltd, Dartford, UK), and confirmed positive results by Western blot (Bio-Rad Laboratories Ltd, Hemel Hempstead, Hertfordshire, UK). Eligible women were randomly assigned in a two-by-two factorial design to receive a daily oral dose of one of four regimens: (1) multivitamins $\left(20 \mathrm{mg} \mathrm{B}, 20 \mathrm{mg} \mathrm{B}, 25 \mathrm{mg} \mathrm{B}_{6}, 100 \mathrm{mg}\right.$ niacin, $50 \mu \mathrm{g} \mathrm{B} \mathrm{B}_{12}, 500 \mathrm{mg}$ vitamin $\mathrm{C}, 30 \mathrm{mg}$ vitamin $\mathrm{E}$ and $0.8 \mathrm{mg}$ folic acid); (2) vitamin A (5000 IU preformed; $1.5 \mathrm{mg}$ ) plus $\beta$-carotene $(30 \mathrm{mg})$; (3) multivitamins including vitamin A plus $\beta$-carotene in doses that were the same as those afore-mentioned; or (4) placebo. The supplements were administered from enrolment throughout the pregnancy and continued after delivery. At delivery, women in groups 1 and 3 received an additional oral dose of vitamin A (200000 IU; $60 \mathrm{mg}$ ), whereas women in groups 2 and 4 were given a placebo. The active treatment and placebo tablets were indistinguishable. Compliance with the study regimens was assessed by pill count. On average, $83 \%$ of participants complied over 2 years from randomisation ${ }^{(13,14)}$. Women and infants received the standard prenatal and child care services in Tanzania. Daily folate and Fe and weekly malaria prophylaxis were provided during pregnancy. All infants received $100000 \mathrm{IU}(30 \mathrm{mg})$ vitamin A at 6 months of age and twice that amount every 6 months thereafter. Antiretroviral therapy was not available in this setting at the time of the study. Breast-feeding was almost universally adopted.

Information on the women's socio-demographic characteristics and obstetric history was obtained at baseline. A study physician performed a complete medical examination, and a study nurse measured the women's weight, height and midupper arm circumference. The stage of HIV disease was determined based on the World Health Organisation Staging System $^{(16)}$. Maternal complete blood counts and absolute $\mathrm{T}$ cell counts were measured at baseline. $\mathrm{CD} 4^{+}$and $\mathrm{CD}^{+}$cell counts were measured using the FACScount system (Becton Dickinson, San Jose, CA, USA). Hb was measured using either a CBC5 Coulter counter (Coulter Corp., Miami, FL, USA) or the cyanmethaemoglobin method with a colorimeter (Corning, Inc., Corning, NY, USA). Plasma levels of vitamins $\mathrm{A}$ and $\mathrm{E}$ were measured using reversed-phase $\mathrm{HPLC}^{(17)}$. At delivery, a research midwife weighed the infants to the nearest $10 \mathrm{~g}$ on a standard beam balance. Gestational age was based on the date of the last menstrual period.

Mortality and morbidity surveillance of children was performed at monthly clinic visits. For women who missed their clinic visits, a home visit was made. We obtained the vital status of women and children from neighbours or relatives. At each clinic visit, mothers were asked about the number of days, if any, that the child had signs of diarrhoeal or respiratory disease during the previous month. Diarrhoea was defined as $\geq 3$ watery stools in the prior $24 \mathrm{~h}$. Acute diarrhoea included all episodes with at least $1 \mathrm{~d}$ but less than $14 \mathrm{~d}$ of diarrhoea. Acute diarrhoea was classified as dysentery, which included all episodes of diarrhoea with mucus or blood, or as watery diarrhoea, which included all other episodes. Episodes of respiratory illness were classified in four ways: the occurrence of cough alone; cough and fever; 'cough plus' (defined as cough with $\geq 1$ of the following signs: difficulty in breathing, chest retractions and refusal to eat, drink or breastfeed); cough with rapid respiratory rate on the day of visit.

For diagnosis of HIV infection in infants, blood samples were collected at birth, at 6 weeks and every 3 months thereafter. A child was determined to be HIV-1 infected if either a peripheral blood mononuclear cell specimen tested positive using a PCR at any point or a plasma specimen obtained at 18 months of age or older tested positive using ELISA and was confirmed by a Western blot test. The serum concentrations of vitamin $B_{12}$ were measured in infants by a competitive magnetic separation assay on the Technicon Immuno-1 analyser $^{(18)}$.

Primary outcomes of interest for the present study were allcause child mortality and HIV infection. Secondary outcomes were birth outcomes and child morbidity. Birth outcomes included stillbirths (delivery of a dead baby at or after 28 weeks' gestation), birth weight, low birth weight (birth weight less than $2500 \mathrm{~g}$ ), preterm birth (delivery before 37 weeks) and small for gestational age (birth weight below 10 th percentile of weight for gestational age $\left.{ }^{(19)}\right)$. Morbidity included diarrhoea and respiratory infections. We also examined the serum concentration of vitamin $\mathrm{B}_{12}$ in children as an outcome.

The present study was conducted according to the guidelines laid down in the Declaration of Helsinki, and the study protocol was approved by the Research and Publications Committee of Muhimbili University of Health and Allied Sciences, the Ethical Committee of the National AIDS Control Program of the Tanzanian Ministry of Health and the Institutional Review Board of the Harvard School of Public Health. Written informed consent was obtained from all participants.

\section{Data analyses}

Of the 1078 HIV-infected women who were enrolled, three were not pregnant, six died before delivery and twenty-seven were lost to follow-up before delivery. Of the remaining 1042 women, seventy-eight pregnancies resulted in fetal deaths (twenty-nine miscarriages and forty-nine stillbirths), twenty-five gave birth to twins and 939 gave birth to singletons. For the analyses, we included forty singleton stillbirths and 919 live singletons with known sex of the infant. Among those singletons, 855 had at least one specimen for HIV testing.

Intent-to-treat analyses were performed. In order to examine the effects of vitamin supplements on birth outcomes by sex, linear regression models were used for birth weight, and binomial regression models with a log link function were used for stillbirths, low birth weight, preterm birth and small for gestational age. We used Cox proportional hazards models to investigate the effects of supplements on all-cause mortality, HIV infection and the combined endpoints of all-cause mortality or HIV infection. We examined the risk 
Table 1. Baseline characteristics of mothers according to treatment assignment (Mean, percentage values and standard deviations)

\begin{tabular}{|c|c|c|c|c|c|c|c|c|c|c|c|c|c|c|c|c|c|c|c|c|c|c|c|c|}
\hline \multirow[b]{4}{*}{ Characteristics } & \multicolumn{12}{|c|}{ Multivitamins } & \multicolumn{12}{|c|}{ Vitamin A plus $\beta$-carotene } \\
\hline & \multicolumn{6}{|c|}{ Girls } & \multicolumn{6}{|c|}{ Boys } & \multicolumn{6}{|c|}{ Girls } & \multicolumn{6}{|c|}{ Boys } \\
\hline & \multicolumn{3}{|c|}{ Yes ( $n$ 241) } & \multicolumn{3}{|c|}{ No (n 228) } & \multicolumn{3}{|c|}{ Yes ( $n$ 241) } & \multicolumn{3}{|c|}{ No $(n 249)$} & \multicolumn{3}{|c|}{ Yes (n 239) } & \multicolumn{3}{|c|}{ No $(n 230)$} & \multicolumn{3}{|c|}{ Yes (n 249) } & \multicolumn{3}{|c|}{ No $(n 241)$} \\
\hline & Mean & SD & $\%$ & Mean & SD & $\%$ & Mean & SD & $\%$ & Mean & SD & $\%$ & Mean & SD & $\%$ & Mean & SD & $\%$ & Mean & SD & $\%$ & Mean & SD & $\%$ \\
\hline $\begin{array}{l}\text { Women's age (years) } \\
\text { Education (\%) }\end{array}$ & 24.9 & 4.7 & & $24 \cdot 5$ & $5 \cdot 0$ & & 24.4 & 4.5 & & $24 \cdot 7$ & 4.8 & & 24.5 & $4 \cdot 6$ & & $25 \cdot 0$ & 5.0 & & 24.9 & 4.9 & & $24 \cdot 2$ & 4.5 & \\
\hline None or adult & & & $7 \cdot 1$ & & & $10 \cdot 1$ & & & 8.7 & & & 5.6 & & & 8.8 & & & 8.3 & & & $5 \cdot 2$ & & & 9.1 \\
\hline $1-4$ years & & & $5 \cdot 8$ & & & 4.4 & & & 4.6 & & & 5.6 & & & 3.4 & & & $7 \cdot 0$ & & & $5 \cdot 2$ & & & $5 \cdot 0$ \\
\hline $5-8$ years & & & 74.3 & & & $75 \cdot 0$ & & & $75 \cdot 1$ & & & 81.5 & & & 74.1 & & & $75 \cdot 2$ & & & 79.9 & & & 76.8 \\
\hline$>8$ years & & & $12 \cdot 9$ & & & $10 \cdot 5$ & & & 11.6 & & & $7 \cdot 2$ & & & $13 \cdot 8$ & & & $9 \cdot 6$ & & & $9 \cdot 6$ & & & 9.1 \\
\hline Nulliparous (\%) & & & 32.5 & & & $36 \cdot 7$ & & & $35 \cdot 3$ & & & 31.8 & & & 37.8 & & & $31 \cdot 1$ & & & $30 \cdot 0$ & & & 37.3 \\
\hline \multicolumn{25}{|c|}{ WHO HIV disease stage (\%) } \\
\hline 1 & & & $80 \cdot 0$ & & & 82.5 & & & $82 \cdot 2$ & & & $76 \cdot 3$ & & & 79.5 & & & 83.0 & & & $78 \cdot 3$ & & & $80 \cdot 1$ \\
\hline II and III & & & $20 \cdot 0$ & & & 17.5 & & & $17 \cdot 8$ & & & 23.7 & & & 20.5 & & & $17 \cdot 0$ & & & $21 \cdot 7$ & & & 19.9 \\
\hline $\begin{array}{l}\mathrm{CD} 4^{+} \text {cell count } \\
\left(\text { cells } / \mathrm{mm}^{3}\right)\end{array}$ & 419 & 192 & & 410 & 202 & & 425 & 203 & & 425 & 212 & & 398 & 179 & & 432 & 212 & & 423 & 214 & & 428 & 201 & \\
\hline $\begin{array}{l}\mathrm{CD}^{+} \text {cell count } \\
\left(\text { cells } / \mathrm{mm}^{3}\right)\end{array}$ & 730 & 307 & & 771 & 330 & & 754 & 332 & & 723 & 326 & & 778 & 322 & & 721 & 313 & & 715 & 304 & & 762 & 353 & \\
\hline $\mathrm{Hb}(\mathrm{g} / \mathrm{l})$ & 93 & 17 & & 95 & 17 & & 94 & 16 & & 96 & 18 & & 94 & 17 & & 94 & 17 & & 94 & 17 & & 96 & 17 & \\
\hline $\begin{array}{l}\text { Plasma vitamin A } \\
(\mu \mathrm{g} / \mathrm{l})\end{array}$ & 243 & 88 & & 245 & 104 & & 245 & 95 & & 257 & 106 & & 235 & 89 & & 252 & 103 & & 252 & 91 & & 249 & 109 & \\
\hline $\begin{array}{l}\text { Plasma vitamin } \mathrm{E} \\
(\mu \mathrm{mol} / \mathrm{l})\end{array}$ & 98 & 34 & & 99 & 31 & & 100 & 25 & & 95 & 29 & & 102 & 32 & & 95 & 32 & & 97 & 26 & & 99 & 28 & \\
\hline $\begin{array}{l}\text { Mid-upper arm } \\
\text { circumference }(\mathrm{cm})\end{array}$ & $25 \cdot 9$ & 2.9 & & $25 \cdot 5$ & $3 \cdot 1$ & & $25 \cdot 5$ & $2 \cdot 8$ & & $25 \cdot 8$ & $2 \cdot 9$ & & $25 \cdot 6$ & $2 \cdot 9$ & & $25 \cdot 7$ & $3 \cdot 0$ & & $25 \cdot 6$ & $2 \cdot 8$ & & $25 \cdot 6$ & $2 \cdot 9$ & \\
\hline Weight $(\mathrm{kg})$ & 57.9 & $9 \cdot 0$ & & 56.7 & 9.6 & & 57.3 & 8.8 & & $57 \cdot 0$ & 8.7 & & 57.3 & 9.5 & & $57 \cdot 3$ & $9 \cdot 1$ & & $57 \cdot 0$ & 8.5 & & $57 \cdot 2$ & 9.0 & \\
\hline BMI $\left(\mathrm{kg} / \mathrm{m}^{2}\right)$ & 23.5 & $3 \cdot 1$ & & $23 \cdot 1$ & 3.5 & & 23.5 & 3.3 & & $23 \cdot 2$ & $3 \cdot 2$ & & 23.3 & $3 \cdot 3$ & & $23 \cdot 3$ & $3 \cdot 3$ & & 23.4 & 3.2 & & 23.3 & 3.3 & \\
\hline
\end{tabular}


Table 2. Effect of maternal multivitamins supplementation on birth outcomes according to sex

\begin{tabular}{|c|c|c|c|c|c|c|c|c|c|c|c|c|c|c|c|}
\hline \multirow[b]{4}{*}{ Endpoint } & \multicolumn{14}{|c|}{ Multivitamins } & \multirow[b]{4}{*}{$P_{\text {int }} \dagger$} \\
\hline & \multicolumn{7}{|c|}{ Girls } & \multicolumn{7}{|c|}{ Boys } & \\
\hline & \multicolumn{2}{|c|}{ Yes } & \multicolumn{2}{|c|}{ No } & \multirow[b]{2}{*}{$\mathrm{RR}^{*}$} & \multirow[b]{2}{*}{$95 \% \mathrm{Cl}$} & \multirow[b]{2}{*}{$P$} & \multicolumn{2}{|c|}{ Yes } & \multicolumn{2}{|c|}{ No } & \multirow[b]{2}{*}{$\mathrm{RR}^{\star}$} & \multirow[b]{2}{*}{$95 \% \mathrm{Cl}$} & \multirow[b]{2}{*}{$P$} & \\
\hline & $N$ & $\%$ & $N$ & $\%$ & & & & $N$ & $\%$ & $N$ & $\%$ & & & & \\
\hline Stillbirth & 5 & $2 \cdot 1$ & 11 & $4 \cdot 8$ & 0.43 & $0.15,1 \cdot 22$ & 0.11 & 7 & 2.9 & 17 & $6 \cdot 8$ & 0.43 & $0.18,1.01$ & 0.05 & 0.99 \\
\hline Low birth weight & 16 & 7.6 & 37 & $19 \cdot 6$ & 0.39 & $0.22,0.67$ & $<0.001$ & 17 & $8 \cdot 1$ & 21 & $10 \cdot 0$ & 0.81 & $0.44,1.49$ & 0.50 & 0.08 \\
\hline Preterm delivery & 53 & 22.5 & 56 & $25 \cdot 8$ & 0.87 & $0.63,1.21$ & 0.41 & 56 & 23.9 & 60 & $25 \cdot 9$ & 0.93 & $0.67,1.27$ & 0.63 & 0.79 \\
\hline Small for gestational age & 19 & 9.0 & 35 & 18.5 & 0.49 & $0.29,0.82$ & 0.007 & 14 & $6 \cdot 6$ & 24 & 11.4 & 0.58 & $0.31,1.10$ & 0.09 & 0.66 \\
\hline
\end{tabular}

$\mathrm{RR}$, relative risks.

${ }^{*} \mathrm{RR}$ and $95 \% \mathrm{Cl}$ are obtained from binomial regression model.

$\dagger P_{\text {int }}$ test for interaction between multivitamins regimen and sex from the likelihood ratio test.

of mortality within the strata of HIV infection as a time-varying covariate by using a counting process data structure. Likelihood ratio tests were used to assess the statistical significance of the interactions by sex and treatment effects. The KaplanMeier method was used to construct survival curves. The effects of supplements on diarrhoea and respiratory infections were examined using generalised estimating equations with an exchangeable working covariance structure. A log link function with a binomial working covariance was used in these models. All statistical analyses were conducted using SAS version 9.1 (SAS Institute, Cary, NC, USA).

\section{Results}

Within strata of sex, treatment groups were comparable with respect to maternal characteristics at baseline (Table 1). The mean age of women was 24.7 (SD 4.7) years at baseline. The majority $(80 \%)$ of pregnant women were in stage I of HIV disease and $12 \%$ had a CD4 ${ }^{+}$cell count $<200$ cells $/ \mathrm{mm}^{3}$.

\section{Effect of vitamin supplements on birth outcomes by sex}

We have previously reported that multivitamin supplements significantly reduced the risks of fetal death and low birth weight $^{(15)}$. Multivitamins decreased the risk of stillbirths non-differentially by sex ( $P$ for interaction between sex and regimen $=0.99$; Table 2 ). The beneficial effect of multivitamins on birth weight was stronger among girls (3051 v. $2893 \mathrm{~g}$; mean difference $=158 \mathrm{~g}$ ) than among boys (3074 $v$. $3033 \mathrm{~g}$; mean difference $=41 \mathrm{~g} ; \quad P$ for interaction $=0 \cdot 10)$. Multivitamin supplementation reduced the incidence of low birth weight by $61 \%$ among girls (relative risks $(\mathrm{RR})=0.39$, $95 \%$ CI $0.22,0.67)$ and by $19 \%$ among boys $(\mathrm{RR}=0.81$, $95 \%$ CI $0.44,1.49 ; P$ for interaction $=0.08$ ). Multivitamins significantly reduced the risk of being small for gestational age among both sexes $(P$ for interaction $=0 \cdot 66$ ). We have previously found no effects of vitamin A plus $\beta$-carotene on fetal death, low birth weight or small for gestational age ${ }^{(15)}$; and we found no differential effect on these birth outcomes by sex.

\section{Effect of vitamin supplements on mortality and HIV infection} by sex

The effect of multivitamin supplements on child mortality among live births by 24 months of age differed by sex ( $P$ for interaction $=0 \cdot 04$; Table 3 ). Multivitamin supplements resulted in $32 \%$ reduction in mortality among girls $(\mathrm{RR}=0.68,95 \%$ CI $0.47,0.97)$, whereas no evidence of

Table 3. Effect of maternal multivitamins supplementation on child mortality and HIV infection according to sex

\begin{tabular}{|c|c|c|c|c|c|c|c|c|c|c|c|c|c|c|c|}
\hline \multirow[b]{4}{*}{ Endpoint } & \multicolumn{14}{|c|}{ Multivitamins } & \multirow[b]{4}{*}{$P_{\text {int }} \dagger$} \\
\hline & \multicolumn{7}{|c|}{ Girls } & \multicolumn{7}{|c|}{ Boys } & \\
\hline & \multicolumn{2}{|c|}{ Yes } & \multicolumn{2}{|c|}{ No } & \multirow[b]{2}{*}{$\mathrm{RR}^{*}$} & \multirow[b]{2}{*}{$95 \% \mathrm{Cl}$} & \multirow[b]{2}{*}{$P$} & \multicolumn{2}{|c|}{ Yes } & \multicolumn{2}{|c|}{ No } & \multirow[b]{2}{*}{$\mathrm{RR}^{*}$} & \multirow[b]{2}{*}{$95 \% \mathrm{Cl}$} & \multirow[b]{2}{*}{$P$} & \\
\hline & $n$ & $N$ & $n$ & $N$ & & & & $n$ & $N$ & $n$ & $N$ & & & & \\
\hline $\begin{array}{l}\text { Mortality including stillbirths } \\
\text { Mortality among live births }\end{array}$ & 59 & 241 & 77 & 228 & 0.64 & $0.46,0.90$ & 0.01 & 60 & 241 & 62 & 249 & 0.99 & $0.69,1.41$ & 0.95 & 0.09 \\
\hline All children & 54 & 236 & 66 & 217 & 0.68 & $0.47,0.97$ & 0.03 & 53 & 234 & 45 & 232 & 1.20 & $0.80,1.78$ & 0.38 & 0.04 \\
\hline HIV negative & 12 & 147 & 19 & 135 & 0.55 & $0.26,1 \cdot 17$ & 0.12 & 18 & 156 & 20 & 164 & 0.80 & $0.41,1.56$ & 0.50 & 0.47 \\
\hline HIV positiveł & 36 & 75 & 32 & 57 & 0.76 & $0.47,1.23$ & 0.27 & 28 & 61 & 22 & 60 & 1.27 & $0.72,2.24$ & 0.41 & 0.16 \\
\hline Total HIV infection§ & 75 & 222 & 57 & 192 & $1 \cdot 16$ & $0.82,1 \cdot 64$ & 0.40 & 61 & 217 & 60 & 224 & 1.05 & $0.73,1.50$ & 0.85 & 0.73 \\
\hline $\begin{array}{l}\text { Total HIV infection or } \\
\text { mortality including } \\
\text { stillbirths }\end{array}$ & 98 & 241 & 102 & 228 & 0.87 & $0.66,1 \cdot 15$ & 0.33 & 93 & 241 & 100 & 249 & 0.95 & $0.71,1.26$ & 0.66 & 0.68 \\
\hline
\end{tabular}

$\mathrm{n}$, number of endpoints; $\mathrm{N}$, number of individuals at risk; RR, relative risks.

${ }^{*} \mathrm{RR}, 95 \% \mathrm{Cl}$ and $P$ values are obtained from Cox proportional hazards model.

$\dagger P_{\text {int }}$ test for interaction between multivitamins regimen and sex from the likelihood ratio test.

$\ddagger$ Time-varying HIV status from birth among live births.

$\S$ Total HIV infection includes intrauterine, intrapartum and breast-feeding transmission. 
(a)

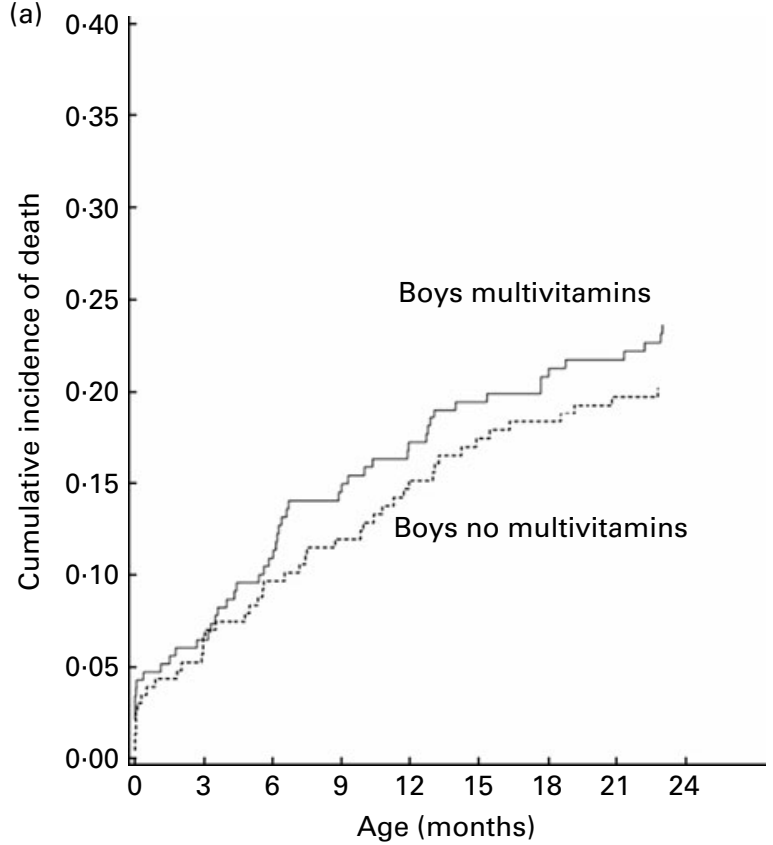

Number at risk

$\begin{array}{llllllll}\text { No MV } & 229 & 212 & 202 & 194 & 186 & 176 & 172 \\ \text { MV } & 228 & 212 & 199 & 189 & 182 & 172 & 160\end{array}$

(b)

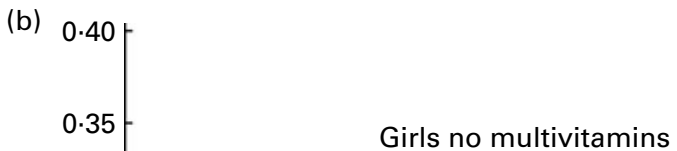

Number at risk

\begin{tabular}{|c|c|c|c|c|c|}
\hline MV & 210 & 183 & 172 & 16 & 15 \\
\hline MV & 233 & 217 & 207 & 19 & \\
\hline
\end{tabular}

Fig. 1. Effect of maternal multivitamin supplementation on child mortality among live births according to sex. (a) Effect of multivitamins (MV) on mortality among girls; (b) effect of multivitamins on mortality among boys.

the effect was found among boys $(\mathrm{RR}=1 \cdot 20,95 \%$ CI $0 \cdot 80$, 1.78; Fig. 1). We observed similar effects when stillbirths were included in the analysis. The effects of multivitamins on the risk of HIV transmission or HIV-free survival did not differ by sex ( $P$ for interaction $=0.73$ and 0.68 , respectively). We have previously found that maternal vitamin A plus $\beta$-carotene supplements had no effect on child mortality by 24 months, but increased the overall risk of HIV transmission $^{(13)}$. We did not observe any differences between boys and girls in the effects of vitamin A plus $\beta$-carotene on mortality or the risk of mother-to-child transmission of HIV ( $P$ for interaction $=0.82$ and $0 \cdot 22$, respectively; Table 4 ).
We also examined the effect of supplements by four treatment arms, and we did not find any interactions between the multivitamins and the vitamin A plus $\beta$-carotene regimens. Compared with placebo, multivitamins alone reduced mortality among girls $(\mathrm{RR}=0.58,95 \%$ CI $0.34,0.97)$, but not among boys $(\mathrm{RR}=1.45,95 \%$ CI $0.81,2.58 ; \quad P$ for interaction $=0.02$ ). Multivitamins together with vitamin A plus $\beta$-carotene also showed sex differential effects (girls $\mathrm{RR}=0.69,95 \%$ CI $0.42,1.14$ and boys $\mathrm{RR}=1.33,95 \%$ CI $0.74,2.39 ; P$ for interaction $=0.09$ ). However, vitamin $\mathrm{A}$ plus $\beta$-carotene supplements showed no effect among girls and boys when compared with placebo (girls $\mathrm{RR}=0 \cdot 88$,

Table 4. Effect of maternal vitamin A plus $\beta$-carotene supplementation on child mortality and HIV infection according to sex

\begin{tabular}{|c|c|c|c|c|c|c|c|c|c|c|c|c|c|c|c|}
\hline \multirow[b]{4}{*}{ Endpoint } & \multicolumn{14}{|c|}{ Vitamin A plus $\beta$-carotene } & \multirow[b]{4}{*}{$P_{\text {int }} \dagger$} \\
\hline & \multicolumn{7}{|c|}{ Girls } & \multicolumn{7}{|c|}{ Boys } & \\
\hline & \multicolumn{2}{|c|}{ Yes } & \multicolumn{2}{|c|}{ No } & \multirow[b]{2}{*}{$\mathrm{RR}^{*}$} & \multirow[b]{2}{*}{$95 \% \mathrm{Cl}$} & \multirow[b]{2}{*}{$P$} & \multicolumn{2}{|c|}{ Yes } & \multicolumn{2}{|c|}{ No } & \multirow[b]{2}{*}{$\mathrm{RR}^{*}$} & \multirow[b]{2}{*}{$95 \% \mathrm{Cl}$} & \multirow[b]{2}{*}{$P$} & \\
\hline & $n$ & $N$ & $n$ & $N$ & & & & $n$ & $N$ & $n$ & $N$ & & & & \\
\hline $\begin{array}{l}\text { Mortality including stillbirths } \\
\text { Mortality among live births }\end{array}$ & 70 & 239 & 66 & 230 & $1 \cdot 00$ & $0.71,1.40$ & 0.98 & 63 & 249 & 59 & 241 & 1.07 & $0.75,1.53$ & 0.71 & 0.76 \\
\hline All children & 63 & 232 & 57 & 221 & 1.03 & $0.72,1.48$ & 0.86 & 51 & 237 & 47 & 229 & $1 \cdot 10$ & $0.74,1.63$ & 0.65 & 0.82 \\
\hline HIV negativeł & 15 & 138 & 16 & 144 & 0.99 & $0.47,2.08$ & 0.98 & 17 & 151 & 21 & 169 & 1.00 & $0.52,1.94$ & 0.99 & 0.99 \\
\hline HIV positiveł & 35 & 73 & 33 & 59 & 0.79 & $0.49,1.28$ & 0.34 & 31 & 74 & 19 & 47 & $1 \cdot 30$ & $0.72,2.32$ & 0.38 & 0.21 \\
\hline Total HIV infection§ & 73 & 211 & 59 & 203 & $1 \cdot 20$ & $0.85,1.69$ & 0.31 & 74 & 225 & 47 & 216 & 1.65 & $1 \cdot 14,2 \cdot 38$ & 0.01 & 0.22 \\
\hline $\begin{array}{l}\text { Total HIV infection or } \\
\text { mortality including stillbirths }\end{array}$ & 108 & 239 & 92 & 230 & $1 \cdot 15$ & $0.87,1.52$ & 0.34 & 106 & 249 & 87 & 241 & 1.27 & $0.95,1.69$ & 0.11 & 0.64 \\
\hline
\end{tabular}

$\mathrm{n}$, number of endpoints; $\mathrm{N}$, number of individuals at risk; RR, relative risks.

${ }^{*} \mathrm{RR}, 95 \% \mathrm{Cl}$ and $P$ values are obtained from Cox proportional hazards model.

$\dagger P_{\text {int }}$ test for interaction between vitamin A plus $\beta$-carotene regimen and sex from the likelihood ratio test.

$\ddagger$ Time-varying HIV status from birth among live births.

$\S$ Total HIV infection includes intrauterine, intrapartum and breast-feeding transmission. 
Table 5. Effect of maternal multivitamins or vitamin A plus $\beta$-carotene supplements on the incidence of diarrhoea and respiratory infection in children according to sex

\begin{tabular}{|c|c|c|c|c|c|c|c|c|c|c|c|c|c|c|}
\hline \multirow[b]{3}{*}{ Endpoint } & \multicolumn{7}{|c|}{ Multivitamins } & \multicolumn{7}{|c|}{ Vitamin A plus $\beta$-carotene } \\
\hline & \multicolumn{3}{|c|}{ Girls } & \multicolumn{3}{|c|}{ Boys } & \multirow[b]{2}{*}{$P_{\text {int }}{ }^{-}$} & \multicolumn{3}{|c|}{ Girls } & \multicolumn{3}{|c|}{ Boys } & \multirow[b]{2}{*}{$P_{\text {int }}{ }^{\dagger}$} \\
\hline & $\mathrm{RR}^{*}$ & $95 \% \mathrm{Cl}$ & $P$ & $\mathrm{RR}^{*}$ & $95 \% \mathrm{Cl}$ & $P$ & & $\mathrm{RR}^{*}$ & $95 \% \mathrm{Cl}$ & $P$ & $\mathrm{RR}^{\star}$ & $95 \% \mathrm{Cl}$ & $P$ & \\
\hline \multicolumn{15}{|l|}{ Diarrhoea } \\
\hline All & 0.91 & $0.74,1.13$ & 0.39 & 0.80 & $0.66,0.97$ & 0.02 & 0.37 & $1 \cdot 11$ & $0.90,1.38$ & 0.32 & 0.85 & $0.71,1.03$ & $0 \cdot 10$ & 0.07 \\
\hline Acute & 0.89 & $0.72,1 \cdot 10$ & 0.30 & 0.81 & $0.67,0.98$ & 0.03 & 0.52 & 1.08 & $0.87,1.34$ & 0.48 & 0.83 & $0.69,1.00$ & 0.06 & 0.07 \\
\hline Watery & 0.91 & $0.68,1 \cdot 21$ & 0.50 & 0.77 & $0.59,1.00$ & 0.05 & 0.40 & $1 \cdot 13$ & $0.84,1.50$ & 0.42 & 0.75 & $0.58,0.97$ & 0.03 & 0.04 \\
\hline Dysenteric & 0.89 & $0.67,1.17$ & 0.39 & 0.86 & $0.66,1 \cdot 13$ & 0.27 & 0.87 & 1.03 & $0.78,1.36$ & 0.82 & 0.89 & $0.68,1.17$ & 0.41 & 0.48 \\
\hline \multicolumn{15}{|l|}{ Respiratory infection } \\
\hline Cough & 1.04 & $0.92,1.17$ & 0.57 & 1.02 & $0.92,1.13$ & 0.75 & 0.74 & $1 \cdot 12$ & $0.99,1.26$ & 0.06 & 0.96 & $0.86,1.07$ & 0.45 & 0.06 \\
\hline Cough and fever & 1.04 & $0.85,1.28$ & 0.70 & 0.99 & $0.82,1.20$ & 0.95 & 0.73 & $1 \cdot 10$ & $0.89,1.35$ & 0.38 & 0.94 & $0.78,1 \cdot 14$ & 0.56 & 0.28 \\
\hline Cough plusł & $1 \cdot 10$ & $0.81,1.50$ & 0.55 & 0.81 & $0.60,1.08$ & 0.15 & 0.15 & $1 \cdot 13$ & $0.83,1.54$ & 0.43 & 0.99 & $0.74,1.33$ & 0.97 & 0.51 \\
\hline $\begin{array}{l}\text { Cough and rapid } \\
\text { respiratory rate }\end{array}$ & 1.36 & $0 \cdot 86,2 \cdot 16$ & 0.19 & 0.97 & $0.60,1.56$ & 0.90 & 0.32 & 0.82 & $0.52,1 \cdot 28$ & 0.38 & 0.62 & $0.38,1.00$ & 0.05 & 0.40 \\
\hline
\end{tabular}

RR, relative risks.

${ }^{*} \mathrm{RR}, 95 \% \mathrm{Cl}$ and $P$ values are obtained from generalised estimating equations using a log link function with a binomial variance assumption.

$\dagger P_{\text {int }}$ test for interaction between regimen and sex from the robust score test.

$\ddagger$ Cough plus defined as cough with $\geq 1$ of the following signs: difficulty in breathing, chest retractions and refusal to eat, drink or breastfeed.

$95 \%$ CI $0 \cdot 54,1 \cdot 43$ and boys $\mathrm{RR}=1 \cdot 33,95 \%$ CI $0 \cdot 74,2 \cdot 40$;

$P$ for interaction $=0 \cdot 29$ ).

\section{Effect of vitamin supplements on morbidity by sex}

We have previously found that multivitamin supplements significantly reduced the overall risks of diarrhoea by $17 \%$, and that they also had beneficial effects on the risks of acute diarrhoea, watery diarrhoea and dysentery ${ }^{(14)}$. These effects on diarrhoea-related endpoints did not significantly differ by sex (Table 5). Multivitamin supplementation had no effect on the risks of respiratory infections, and there were no interactions between the multivitamins regimen and sex. We have previously reported no effect of vitamin A plus $\beta$-carotene on the risks of diarrhoea-associated outcomes ${ }^{(14)}$. However, boys may have benefited from vitamin A plus $\beta$-carotene with regard to acute diarrhoea and watery diarrhoea ( $P$ for interaction $=0.07$ and 0.04 , respectively; Table 5). We have previously found that supplementation with vitamin A plus $\beta$-carotene reduced the risk of cough with a rapid respiratory rate, a proxy for pneumonia, but no effects were observed for other respiratory endpoints ${ }^{(14)}$. We found no sex differences in the effects on respiratory infections.

\section{Effect of vitamin supplements on micronutrient status by sex}

We have previously reported that maternal multivitamin supplements increased serum concentrations of vitamin $B_{12}$ in infants at 6 weeks and 6 months ${ }^{(18)}$. The beneficial effects that were observed were similar among girls and boys. At 6 weeks, serum vitamin $B_{12}$ was $185 \mathrm{pmol} / \mathrm{l}$ higher among girls with maternal multivitamins (441 (SD 190) pmol/l) than among girls without maternal multivitamins (256 (SD 107) pmol/l). Similarly, serum vitamin $B_{12}$ was $166 \mathrm{pmol} / 1$ higher among boys with multivitamins (406 (SD 183) pmol/l) than among boys without multivitamins (240 (SD 98) pmol/l). These beneficial effects of multivitamin supplements on the concentration of vitamin $B_{12}$ were sustained through 6 months (mean difference $=150 \mathrm{pmol} / \mathrm{l}$ among girls and $105 \mathrm{pmol} / \mathrm{l}$ among boys).

\section{Discussion}

Supplementation of HIV-infected women during pregnancy and lactation with vitamins $\mathrm{B}$-complex, $\mathrm{C}$ and $\mathrm{E}$ at multiples of the RDA reduced the risk of mortality among girls by $32 \%$, but no beneficial effect was observed among boys. Multivitamins had beneficial effects on the risk of diarrhoea, and the beneficial effects did not differ by sex. Vitamin A plus $\beta$-carotene alone increased the risk of HIV transmission but had no effects on mortality, and we found no sex differences in these effects.

The present study did not find sex differences in the effect of vitamin A plus $\beta$-carotene supplements on mortality. However, we found that vitamin A plus $\beta$-carotene lowered the risks of diarrhoea among boys. Previous trials demonstrated that vitamin A supplements reduced the severity of diarrhoeal disease, which may be due to the mediation of vitamin A on strengthening the integrity of mucosal epithelia in the gut and improving the immune function ${ }^{(2)}$. The present findings conflict with previous evidence suggesting that neonatal vitamin A supplements may be associated with a reduced mortality only in boys, but it is consistent with regard to diarrhoea morbidity ${ }^{(5)}$. Benn et al. ${ }^{(11)}$ hypothesised that vitamin A may interact with routine childhood vaccinations; the beneficial effect of vitamin A may be weakened by diphtheria-pertussis-tetanus vaccination, especially for girls. Most infants were vaccinated with diphtheria-pertussis-tetanus in the present study, and we did not observe any differences in the effect of vitamin A plus $\beta$-carotene on mortality. In contrast to previous trials, the present study consisted of children of HIV-infected mothers. Furthermore, mothers received daily vitamin A plus $\beta$-carotene supplements in the present study, whereas in other studies, young infants received a large dose of vitamin A at birth or at the time of routine vaccinations. Vitamin A supplementation to HIV-infected pregnant women should be avoided because of the increased risk of HIV transmission observed in the present study and in Zimbabwe ${ }^{(13,20)}$.

The beneficial effect of multivitamin supplements on reducing the risk of low birth weight was stronger among girls than among boys, and this suggests that sex differential 
effects of multivitamins may have started during pregnancy. Previous trials also found evidence that was consistent with the present results. The effect of micronutrient supplements on birth weight gain was stronger among girls born to presumably HIV-negative women in Nepal (girls, $108 \mathrm{~g} v$. boys, $44 \mathrm{~g}$ ), and birth size gain was stronger among girls in Zimbabwe (girls, $0.5 \mathrm{~cm} v$. boys, $0.02 \mathrm{~cm})^{(21,22)}$. A recent large trial has showed that multivitamins reduced the incidence of low birth weight among HIV-negative women in Tanzania ${ }^{(23)}$. In this trial, girls had gained slightly more weight at birth than boys (girls, $72 \mathrm{~g} v$. boys, $54 \mathrm{~g}$; unpublished results).

Several possible mechanisms may explain sex differential effects of multivitamins on pregnancy outcomes and survival. The thymus plays a central role in the development of $\mathrm{T}$ lymphocytes. HIV-infected infants often have their thymus disrupted by $\mathrm{HIV}^{(24)}$. A small thymus size at birth is associated with impaired cellular immunity and malnutrition, and it is a strong risk factor for infant mortality ${ }^{(25,26)}$. Girls have smaller thymus indices than boys ${ }^{(25)}$. Girls may be more susceptible to maternal malnutrition and may benefit from multivitamins more than boys during fetal development. In the present study, low-birth weight girls were more prevalent than boys in the groups that did not receive multivitamins, and multivitamins decreased the risk of low birth weight by $61 \%$ among girls and by $19 \%$ among boys. Thymus size and thymic hormone activity are reduced among low-birth weight infants ${ }^{(25,27)}$. Particularly among children born to HIV-infected mothers, low birth weight is a strong risk factor for infant mortality ${ }^{(28)}$. The differences in the effect of multivitamin supplements on infant girls compared with boys may be due to their varying immune systems. Infants primarily have $\mathrm{Th} 2$ immune response at birth and later shift into Th1 response ${ }^{(29,30)}$. The balance between Th1 and Th2 responses is important, and the response biased towards Th2 may increase the risk of infection and mortality during infancy. Supplementations with vitamins $\mathrm{B}_{6}, \mathrm{C}$ and $\mathrm{E}$ alter the Th2 immune response to a proinflammatory Th1 cytokine-regulated response with enhanced innate immunity ${ }^{(31,32)}$. The beneficial effect of multivitamins may have been stronger among girls than among boys because girls have predominantly Th2-mediated immunity ${ }^{(33-35)}$. Evidence shows that girls tend to have excess mortality from Th2 response-exacerbating infectious diseases, whereas boys from Th1 response-exacerbating diseases ${ }^{(33)}$. Female sex hormones may suppress immune responses and affect susceptibility to infections ${ }^{(34)}$. Other possible explanations are hormone-mediated differences in metabolism or genetic differences by sex.

Little is known about sex-related differences in the effects of vitamins on nutritional status of infants. Because the present study found that multivitamin supplements had similar effects on improving vitamin $\mathrm{B}_{12}$ status in girls and boys, sex differences in the immunological or genetic factors may have led to differential effects of multivitamins on survival.

In conclusion, maternal multivitamin (vitamins B-complex, $\mathrm{C}$ and $\mathrm{E})$ supplements reduced the risk of mortality among girls but not among boys born to HIV-infected women. Even though we observed sex differential effects on child mortality, multivitamins are recommended to all HIV-infected pregnant and lactating women because of the beneficial effects among mothers on delaying HIV disease progression ${ }^{(36)}$.
Antiretroviral therapy was not available when the trial was conducted. Therefore, we may not be able to generalise the present results to mothers who received antiretroviral therapy. Furthermore, the present findings may not be generalisable among children of HIV-negative women. More research is warranted to examine the effects of vitamin supplements by sex and elucidate the biological mechanisms of sex differential effects on morbidity and mortality.

\section{Acknowledgements}

The present work was supported by the National Institute of Child Health and Human Development (NICHD R01 32257) and the Fogarty International Center (NIH D43 TW00004). We thank the mothers and children who participated in the present study and the field teams, including nurses, midwives, supervisors, laboratory staff and administrative staff. K. K. conducted the data analyses, interpreted the results and wrote the initial draft of the manuscript. G. M. and K. M. participated in the study implementation and supervision in the field. E. V., R. J. B. and E. H. participated in the data analyses and the data interpretation. W. W. F. is the principal investigator and contributed to the study design, implemented the study, and analysed and interpreted the data. None of the authors have any conflicts of interest.

\section{References}

1. Fawzi WW, Chalmers TC, Herrera MG, et al. (1993) Vitamin A supplementation and child mortality. A meta-analysis. JAMA 269, 898-903.

2. Villamor E \& Fawzi WW (2000) Vitamin A supplementation: implications for morbidity and mortality in children. $J$ Infect Dis 182, Suppl. 1, S122-S133.

3. Bhutta ZA, Ahmed T, Black RE, et al. (2008) What works? Interventions for maternal and child undernutrition and survival. Lancet 371, 417-440.

4. Gogia S \& Sachdev HS (2009) Neonatal vitamin A supplementation for prevention of mortality and morbidity in infancy: systematic review of randomised controlled trials. BMJ 338, b919.

5. Benn CS, Fisker AB, Diness BR, et al. (2006) Neonatal vitamin a supplementation: sex-differential effects on mortality? J Infect Dis 194, 719.

6. Humphrey JH, Agoestina T, Wu L, et al. (1996) Impact of neonatal vitamin A supplementation on infant morbidity and mortality. J Pediatr 128, 489-496.

7. Rahmathullah L, Tielsch JM, Thulasiraj RD, et al. (2003) Impact of supplementing newborn infants with vitamin A on early infant mortality: community based randomised trial in southern India. BMJ 327, 254.

8. Benn CS, Diness BR, Roth A, et al. (2008) Effect of 50,000 IU vitamin A given with BCG vaccine on mortality in infants in Guinea-Bissau: randomised placebo controlled trial. BMJ 336, 1416-1420.

9. Benn CS, Martins C, Rodrigues A, et al. (2005) Randomised study of effect of different doses of vitamin A on childhood morbidity and mortality. BMJ 331, 1428-1432.

10. Yassai MB \& Malek F (1989) Newborns vitamin A in relation to sex and birth weight. J Trop Pediatr 35, 247-249.

11. Benn CS, Bale C, Sommerfelt H, et al. (2003) Hypothesis: vitamin A supplementation and childhood mortality: amplification of the non-specific effects of vaccines? Int J Epidemiol 32, 822-828. 
12. UNAIDS (2008) Report on the Global AIDS Epidemic. Geneva: UNAIDS.

13. Fawzi WW, Msamanga GI, Hunter D, et al. (2002) Randomized trial of vitamin supplements in relation to transmission of HIV-1 through breastfeeding and early child mortality. AIDS 16, 1935-1944.

14. Fawzi WW, Msamanga GI, Wei R, et al. (2003) Effect of providing vitamin supplements to human immunodeficiency virus-infected, lactating mothers on the child's morbidity and $\mathrm{CD}^{+}$cell counts. Clin Infect Dis 36, 1053-1062.

15. Fawzi WW, Msamanga GI, Spiegelman D, et al. (1998) Randomised trial of effects of vitamin supplements on pregnancy outcomes and $\mathrm{T}$ cell counts in HIV-1-infected women in Tanzania. Lancet 351, 1477-1482.

16. World Health Organization (1993) Proposed 'World Health Organization staging system for HIV infection and disease': preliminary testing by an international collaborative crosssectional study. The WHO International Collaborating Group for the Study of the WHO Staging System. AIDS 7, 711-718.

17. De Leenheer AP, De Bevere VO, De Ruyter MG, et al. (1979) Simultaneous determination of retinol and alpha-tocopherol in human serum by high-performance liquid chromatography. J Chromatogr 162, 408-413.

18. Baylin A, Villamor E, Rifai N, et al. (2005) Effect of vitamin supplementation to HIV-infected pregnant women on the micronutrient status of their infants. Eur J Clin Nutr 59, 960-968.

19. Brenner WE, Edelman DA \& Hendricks CH (1976) A standard of fetal growth for the United States of America. Am J Obstet Gynecol 126, 555-564.

20. Humphrey JH, Iliff PJ, Marinda ET, et al. (2006) Effects of a single large dose of vitamin A, given during the postpartum period to HIV-positive women and their infants, on child HIV infection, HIV-free survival, and mortality. $J$ Infect Dis 193, $860-871$

21. Osrin D, Vaidya A, Shrestha Y, et al. (2005) Effects of antenatal multiple micronutrient supplementation on birthweight and gestational duration in Nepal: double-blind, randomised controlled trial. Lancet 365, 955-962.

22. Friis H, Gomo E, Nyazema N, et al. (2004) Effect of multimicronutrient supplementation on gestational length and birth size: a randomized, placebo-controlled, double-blind effectiveness trial in Zimbabwe. Am J Clin Nutr 80, 178-184.
23. Fawzi WW, Msamanga GI, Urassa W, et al. (2007) Vitamins and perinatal outcomes among HIV-negative women in Tanzania. N Engl J Med 356, 1423-1431.

24. Kourtis AP, Ibegbu C, Nahmias AJ, et al. (1996) Early progression of disease in HIV-infected infants with thymus dysfunction. N Engl J Med 335, 1431-1436.

25. Aaby P, Marx C, Trautner S, et al. (2002) Thymus size at birth is associated with infant mortality: a community study from Guinea-Bissau. Acta Paediatr 91, 698-703.

26. Garly ML, Trautner SL, Marx C, et al. (2008) Thymus size at 6 months of age and subsequent child mortality. $J$ Pediatr 153, 683-688.

27. Chandra RK (1981) Serum thymic hormone activity and cell-mediated immunity in healthy neonates, preterm infants, and small-for-gestational age infants. Pediatrics 67, 407-411.

28. Wei R, Msamanga GI, Spiegelman D, et al. (2004) Association between low birth weight and infant mortality in children born to human immunodeficiency virus 1 -infected mothers in Tanzania. Pediatr Infect Dis $J$ 23, 530-535.

29. Lee HH, Hoeman CM, Hardaway JC, et al. (2008) Delayed maturation of an IL-12-producing dendritic cell subset explains the early Th2 bias in neonatal immunity. J Exp Med 205, $2269-2280$.

30. Levy O (2007) Innate immunity of the newborn: basic mechanisms and clinical correlates. Nat Rev Immunol 7, 379-390.

31. Wintergerst ES, Maggini S \& Hornig DH (2007) Contribution of selected vitamins and trace elements to immune function. Ann Nutr Metab 51, 301-323.

32. Long KZ \& Santos JI (1999) Vitamins and the regulation of the immune response. Pediatr Infect Dis J 18, 283-290.

33. Garenne M \& Lafon M (1998) Sexist diseases. Perspect Biol Med 41, 176-189.

34. Verthelyi D (2001) Sex hormones as immunomodulators in health and disease. Int Immunopharmacol 1, 983-993.

35. Beagley KW \& Gockel CM (2003) Regulation of innate and adaptive immunity by the female sex hormones oestradiol and progesterone. FEMS Immunol Med Microbiol 38, 13-22.

36. Fawzi WW, Msamanga GI, Spiegelman D, et al. (2004) A randomized trial of multivitamin supplements and HIV disease progression and mortality. $N$ Engl J Med 351, $23-32$. 